\title{
Application of Optimization-based Worst Case Analysis to Control Law Assessment in Aerospace
}

\author{
Hans-Dieter Joos ${ }^{1}$
}

\begin{abstract}
The flight control law design assessment problem can be formulated as a robustness analysis problem, where a set of suitably defined assessment criteria must be checked to lie within certain limits for all admissible variations of vehicle parameters, external inputs and all flight conditions. Optimization based worst case analysis can be used to find those parameters/inputs/flight conditions for which the criteria are violated or poorly satisfied. The potential of this approach is its general applicability to any kind of simulation models and scenarios including complex non-linearity in control laws. But in order to confidently assert that no violation of assessment criteria exists, a global optimization problem has to be solved. Especially in case of many assessment criteria, global worst case search can lead to a huge computational effort. However, solving worst case problems as a multi-objective problem can help to reduce the number of computations since all or some of the assessment criteria can be considered simultaneously. Optimization-based approaches can also be used to detect parameter sensitivities on the assessment criteria and can help to find safe parameter regions.
\end{abstract}

\section{Introduction}

When applying traditional approaches like Monte-Carlo simulations or parameter studies for flight control system (FCS) assessment it is problematic to find confidently weak or even worst cases or to confidently assert that those cases do not exist [14]. Along with the normally huge computational effort necessary for these approaches, this motivated research in [13] to explore the benefits of several new analysis techniques for the assessment analysis of flight control laws, like stability analysis of linear parameter varying systems or optimization based worst case search.

In the latter approach, the assessment problem is formulated as a robustness analysis problem, where a set of suitably defined assessment criteria must be checked to lie above (below) certain bounds for all admissible variations of aircraft parameters, external inputs and all flight conditions. Optimization tools can

\footnotetext{
${ }^{1}$ Hans-Dieter Joos

German Aerospace Center (DLR), Institute of Robotics and Mechatronics, 82234 Wessling, Germany, Email: dieter.joos@dlr.de
} 
then be used to find the minimum (maximum) solution indicating whether these bounds are violated or not and the global extremum found is called the worst case. Such an approach was already formulated in [2].

Hence the difficulty in using the optimization based approach does not lie in finding a parameter combination such that a criterion is not satisfied, i.e. to demonstrate that the FCS is not validated, but to confidently assert that a criterion is verified in all cases. That means one has to solve a global optimization problem and the global solution should be found with some reasonable confidence level. A strategy for approaching this is proposed in [12].

There exist several investigations on optimization based assessment regarding different applications and usage of different single objective optimization algorithms, see e.g. $[4,7,9,10,11,14]$. The results clearly demonstrate the high potential of the optimization-based approach in reliably solving assessment problems with many simultaneous uncertain parameters and criteria. The reasons for that are in particular the generality and reliability of the approach without adding conservatism. These advantages also hold for the multi-criteria approach. Since the assessment process normally requires demonstrating system performance for a whole set of criteria one can expect that the multi-objective approach will reduce computational effort substantially since only one optimization run is necessary to get the required results for all criteria under consideration.

Not only worst-case problems can be solved by means of optimization but also the effect of individual parameter variations on assessment criteria violation can be examined. For this a norm optimization problem has to be formulated with the assessment conditions as constraints.

Moreover using the niching concepts introduced for genetic algorithms [5], regions in the parameter space can be detected where assessment criteria are violated or the system is of weak performance. These parameter regions may be spread over the whole admissible parameter range not only concentrated on the region of convergence to the worst case parameter combination.

In this paper the formulation of assessment and sensitivity problems as optimization problems will be given in sect. 2 . The control law assessment process based on this optimization tasks is demonstrated by two aerospace applications. In sect. 3 , multi-objective optimization is used to validate eight control law performance criteria of a nonlinear six degrees of freedom time domain simulation model of the VEGA launcher [8]. For this application also the effects of the more than eighty parameters are investigated applying the proposed optimization approaches. The second application is to find regions of reduced performance in the flight envelope of a research FCS for a large civil transport aircraft regarding load limits exceedance (Sect. 4). The development of a fault tolerant FCS including protections is one of the benchmark problems formulated in the FP7 Project RECONFIGURE (Reconfiguration of Control in Flight for Integral Global Upset Recovery) [15]. 


\section{Formulation of assessment problems as optimization tasks}

\subsection{Worst case search}

Following the approaches in [2, 4, 7, 9, 10, 11, 13] for utilising optimisation methods the assessment problem has to be expressed as a scalar objective function $c(p, d)$ with optimisation parameters $p$ that are uncertain or varying during operation (e.g. aerodynamic coefficients, aircraft mass, inertia, speed, height or wind parameters, etc.) and discrete conditions $d$ (e.g. aircraft configuration, landing gear settings, etc.). The components of $p$ are assumed to be bounded and continuously varying over known intervals, defining a hyper-box $P$. The assessment problem can now be formulated as a minimization problem. Let $c_{0}(d)$ a lower acceptable value of $c$ then

$$
c_{\min }(d)=\min _{p \in P}(c(p, d))
$$

is a measure for the assessment performance. The assessment requirement is fulfilled for condition $d$ if $c_{\min }(d) \geq c_{0}(d)$, otherwise the criterion is not cleared.

This formulation can immediately be extended to several criteria using the theory of Pareto optimality $[16,17]$. The following optimization problem has now to be solved

$\operatorname{minimize}\left[c_{i}(p)\right]$ for $i=1, \ldots, m$, s.t. $p_{j}^{L} \leq p_{j} \leq p_{j}^{U}, j=1, \ldots n$.

If $\left[c_{i}^{*}\left(p^{*}\right)\right], p^{*} \in P_{\text {Pareto }}$ is a solution point of the Pareto-optimal set, the worst case of criterion $c_{i}^{w c}$ is given by the minimum over all $p^{*}$ :

$$
c_{i}^{w c}=\min _{p^{*} \in P_{\text {Pareto }}}\left(c_{i}^{*}\left(p^{*}\right)\right) .
$$

Generally the two most common approaches to solve multiple objective problems are: (1) combine them into a single objective function and obtain a single solution such as in the cases of the weighted sum method or maximum utility functions, or (2) obtain a set of non-dominated Pareto-optimal solutions directly. The purpose of this study is to demonstrate the usefulness of approach (2) for worst case search problems, where only the extreme values of the Pareto-optimal solution set are of primary interest.

A sophisticated algorithm called NSGA-II based on non-dominated sorting was published by Deb [1]. The objective of the NSGA algorithm is to improve the fit of candidate solutions to a Pareto front with respect to a set of objective functions based on the concept of Pareto-ranking [3] where the fitness of an individual 
is measured by the number of individuals which dominate it. An individual (set of parameters) $A$ is dominated by an individual $B$ if the all criteria values corresponding to $B$ are less than the criteria values corresponding to $A$. The Pareto-rank of an individual is then defined as the number of dominating individuals plus one.

The algorithm itself is an instance of an evolutionary algorithm using evolutionary operators including selection, genetic crossover, and genetic mutation. The population is sorted into a hierarchy of sub-populations based on Pareto ranking. The diversity of the members along a Pareto-front is improved by fitness sharing concepts.

The computations of the assessment-study of this paper have been performed by means of DLR's optimization environment MOPS [6]. The algorithm for multiobjective optimization implemented there is a combination of a global exploration mechanism based on non-dominated sorting with a local search algorithm based on gradient-free pattern search. In such a hybrid approach global multi-objective search alternates iteratively with short local single-objective optimization. The following advantages can be expected:

- A deterministic search for each single criterion can provide rapidly good solutions marking the minimum solutions of each criterion.

- Improved minima of the individual objectives widen the range of the Pareto-front. This normally speeds up the search of elements of the Paretofront between these points by means of genetic algorithms.

- The minimum value for each criterion is found more precise by means of local search algorithms. This is especially useful for worst case search.

Experiences showed that a multi-objective WCS costs about the same amount on criteria evaluations as a WCS with a single objective only especially when using the hybrid optimization technique. Hence in case of usually several assessment criteria multi-objective WCS can help to reduce computational effort since all or at least some of the assessment criteria can be considered simultaneously in one run.

\subsection{Regions of compliance}

The problem of finding regions of compliance is of great interest in aerospace in order to get information where a flight envelope should be restricted. In practice, one has to find ranges in the parameter space where system performance is confidently satisfied is of great interest in aerospace in order to get information where a flight envelope should be restricted. A general formulation of this problem at least for simply connected parameter ranges as an optimization problem is:

$$
\max \left[p_{i}^{U}-p_{i}^{L}\right], \text { s.t. } c_{j}(p) \geq c_{j}^{0}, \text { for all } p \in\left[p^{L}, p^{U}\right]
$$


A multi-objective optimization problem has to be solved with the length of parameter intervals as optimization criteria subject to the constraint that the assessment criteria are satisfied. In general this global optimization problem cannot be solved with reasonable effort at least for many parameters.

Without loss of generality we can assume that the optimization parameters are normalized to $[-1,1]$. Restricting now the range of compliance problem to search for centered equal bounded parameter intervals the following practical formulation of the optimization problem can be made assuming that for $\bar{p}=0$ all assessment criteria are satisfied:

$$
\max \left(\|\bar{p}\|_{\infty}\right), \text { s.t. } c_{j} \geq c_{j}^{0}, \bar{p} \ni[-1,1]
$$

Now a single objective optimization has to be solved under the constraint that the assessment criteria are satisfied.

Evolutionary computation offers another concept to find regions of noncompliance, i.e. regions where at least one assessment criterion might be not satisfied. These regions may be disconnectedly spread over the entire parameter range. With classical optimization techniques, which normally converge in some way to the optimal solution, disconnected parameter regions with weak performance cannot be detected safely. The niching (or crowding distance) concepts developed for genetic algorithms allow (i) to keep multiple, highly fit, but significantly different solutions in a generation, and (ii) help to avoid premature convergence to local minima [5].

When niching is applied, the fitness of an individual is calculated as a properly scaled ratio of the objective function value and minimal distance to other individuals in the parameter space. In our optimization environment niching is applied only for single objective problems.

\subsection{Parameter effects on criteria}

The knowledge of the parameter effectivity on the assessment criteria is of great interest for the design of robust control laws. The importance of individual parameters for cases where assessment criteria are violated can be estimated by means of the following optimization problem:

$$
\min \left(\|\bar{p}\|_{1}\right), \text { s.t. } c_{j}<c_{j}^{0}, \bar{p} \ni[-1,1]
$$

Again it is assumed that for zero parameter values all assessment criteria are satisfied. Since all components of parameter vector $\bar{p}$ contribute to the 1-norm, a minimum can only be achieved when all values of ineffective or less effective parameters tend to zero. Due to the constraints, the more effective parameters 
achieve nonzero values. The minimum must not be unique. At the global minimum all ineffective parameters are expected to be zero.

\section{Application control law assessment of a launcher vehicle}

The assessment analysis performed in this study is based on a standard industrial nonlinear, six degree of freedom simulation model of a launch vehicle which was also the benchmark model in the ESA project "Robust Flight Control System Design Verification and Validation Framework” (ESA AO/1-6322/09/NL/JK). The model describes the VEGA launcher, a new European small launch vehicle developed under the responsibility of ESA, including non-linear actuator dynamics, flight mechanics, aerodynamics, wind input, inertia system and sensors. It is equipped with a full guidance navigation and control system for thrust vector control (TVC) and roll and attitude control (RACS). For assessment studies the model is provided as a black-box simulation model allowing no access to structure and states.

However, the model allows external access to a large number of uncertain parameters regarding aerodynamics, wind, inertial reference system, thrust scattering, mass, center of gravity, inertia, thrust offset and misalignment, atmosphere and separation. In this study the first flight phase with height between $35 \mathrm{~m}$ and $60 \mathrm{~km}$ is considered, utilizing 84 uncertainty parameters for assessment. All parameters are scaled to $[-1,1]$ for external access.

The assessment criteria considered here represent the traditional requirements for TVC and RACS during first flight phase. Eight optimization criteria have been extracted from the compliance matrix regarding lateral control performance and load requirements during flight as well as separation conditions:

- Load requirements are validated via the product of dynamic pressure and angle of attack, $Q \alpha$, depending on Mach (criterion C1)

- $\quad$ Drift of position and speed in $y$ - and z-direction are measured within the entire altitude range of $35 \mathrm{~m}$ to $60 \mathrm{~km}$ (criteria C2, C3, C4, and C5).

- Limits on transversal angular acceleration have to be kept during the whole flight phase (criterion C6)

- $\quad$ Transverse angular velocity at separation must be within some limits (criterion C7)

- $\quad$ Total angle of attack at separation (criterion C8)

All criteria are normalized to their limits or envelop bounds such that a violation of the requirement occurs when the criteria values are less than minus one. For a given set of uncertain parameters the first phase of the flight is simulated until separation. The criteria are then calculated from the various outputs provided by the high-fidelity simulation model.

The applicability of multi-objective optimization to such a multi-criteria assessment problem has already been demonstrated in [12]. As a consequence of 
these results the control law has been retuned in order to get better performance especially for loads criteria C1 making the worst case search much more ambitious even though hybrid optimization techniques have been used now.

Fig. 1 shows the criteria results of one optimization run for the parameter combinations with Pareto rank 1, i.e. those points which are candidate for the Pareto front. It shows that all criteria have improved performance compared to the control law assessed in [12]. However, there are still violations of the assessment bound of -1 for criteria 1 and 7 . Moreover it can be seen that there exist parameter combinations where both criteria are violated at the same time.

Following the assessment strategy outlined in [12] to assert that the global worst cases are reached reliably the WCS has been repeated 10 times with different initial and statistical conditions. Applying 10 runs and assuming a success rate of 0.7 for sufficiently approaching the (global) worst cases by one optimization run a confidence level of $99 \%$ is achieved.

In all runs no WCS could violate criteria C2 to C6 which therefore can be considered as verified. However, criteria C1, C7 and C8 are violating the assessment bounds at least slightly (C8) in all runs. For these criteria the assessment requirements are therefore still not fulfilled.

The hybrid optimization was performed applying a population size of 200 over 100 generations, yielding 20000 simulations and criteria evaluations for global search only. The iteratively activated local searches of the hybrid algorithm require on average almost the same amount on evaluations. Such an optimization run with approximately 40000 evaluations takes about 4 hours of computation time on a desktop PC equipped with two Intel ${ }^{\circledR}$ Xeon ${ }^{\circledR}$ Processors X5550 (2.66GHz) when the parallelization features of MOPS were used utilizing 4 workers.

For the overall assessment problem with 8 criteria and 84 uncertain parameters a region of non-compliance was looked for by means of the norm optimization problem formulated by (5). The max-norm optimization results in the maximum parameter interval around zero, normally the nominal case, inside which the assessment criteria are not violated for all parameter combinations, provided that the global minimum was found. Fig. 2 shows the result of an exhaustive optimization run with about 57.000 evaluations, using the genetic optimization method implemented in MOPS. The blue bars indicate that parameters are allowed to deviate up to about $75 \%$ from nominal before a violation of any of the assessment criteria occurs.

Parameter sensitivity was investigated by a follow on optimization run now minimizing the 1-norm of the uncertainties where the parameter range is restricted to the maximum magnitude of 0.75 as found before. It is expected that all parameters which have no or only less effect on the criteria will tend to zero. The result is shown by the green bars. Several parameters represented by a large blue bar are not accompanied by green ones; see for example parameters numbered as 19 and 21. This indicates that these parameters have no or only small effect on criteria violations. However, since the parameter region is restricted, a deviation of $75 \%$ 
from nominal is still necessary for more than 30 parameters in order to violate at least one criterion.

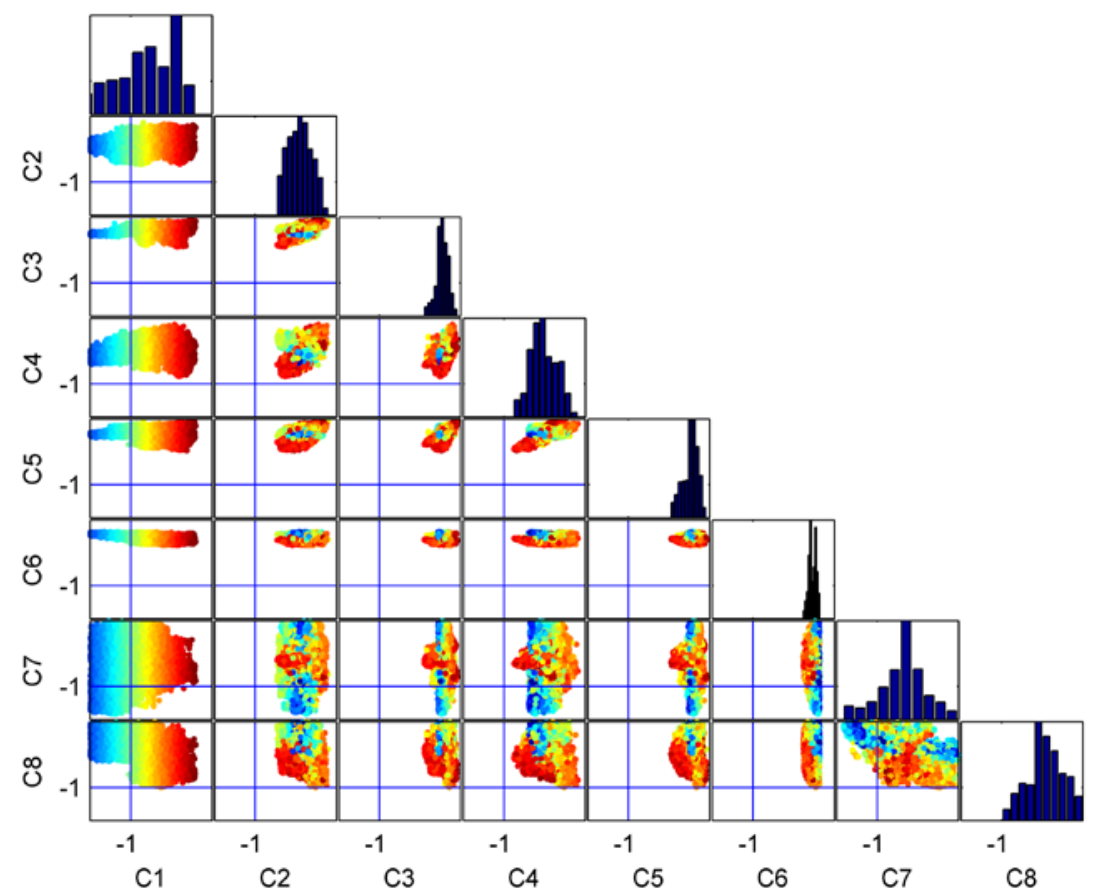

Fig. 1: Two-dimensional section planes of the 8-dimensional Pareto-front for all criteria pairs. The diagrams in the diagonal show the histogram of a single criterion.

When deviations of the whole parameter range are allowed, the optimization problem (6) gives an answer to the question which parameters are the most sensitive ones. An example result of such an optimization is depicted by the brown bars in Fig. 2. Only 10 parameters show a significant deviation from nominal. Hence parameter deviations of those 10 most sensitive parameters will result in violations of at least one assessment criterion. However, the deviation must be up to $100 \%$ for most of the parameters. 


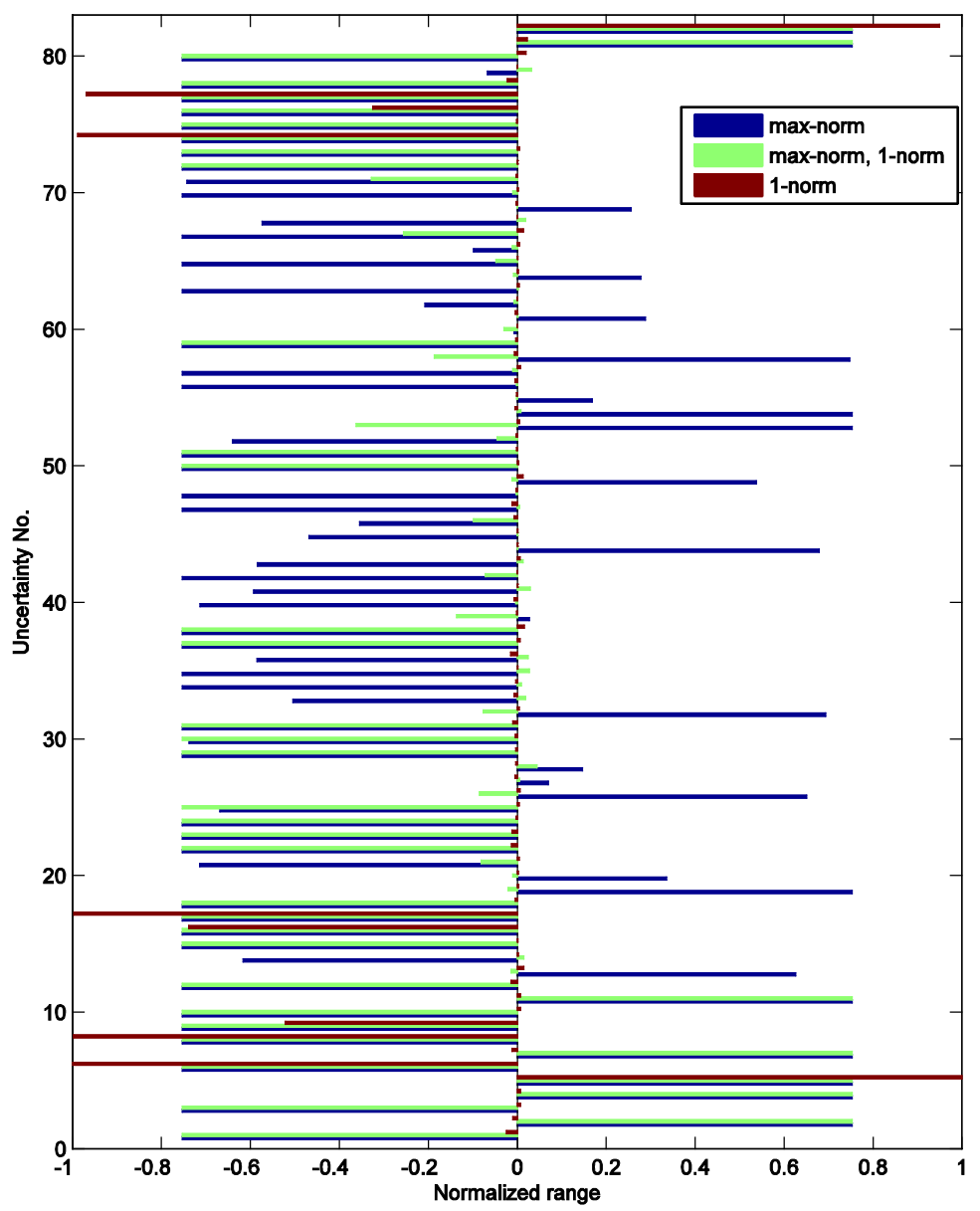

Fig. 2: Range of non-compliance found by max-norm optimization and parameter sensitivity found by 1-norm optimization for the launcher vehicle.

\section{Application of the niching concepts to detect regions of non- compliance}

For a large commercial aircraft one of the assessment requirements is that normal load should not exceed $2.5 \mathrm{~g}$. This property is validated using a high-fidelity industrial aircraft simulation model which is augmented by an experimental FCS including non-linear protection laws to prevent load factor exceedance and to comply with angle of attack, attitude angle or speed limits. WCS is able to keep with 
such a highly non-linear assessment problem without approximations or simplifications.

The diagrams in Fig. 3 and Fig. 4 show the results of two different WC searches. Both diagrams depict the points visited during optimization in the normalized flight domain. Points that violate load factor assessment bounds are marked with a red cross. About 200 evaluations have been performed for each optimization run. Fig. 3 shows the search result without using niching concepts. The optimizer converges rapidly to a minimum indicating bad performance in a single region around the normalized altitude of 0.6 and high speed. In Fig. 4 where the results with niching are depicted a second region of weak performance can be recognized (normalized altitude about 0 ). However, the minimum found by the run applying niching concepts is not as small as the one found without niching.

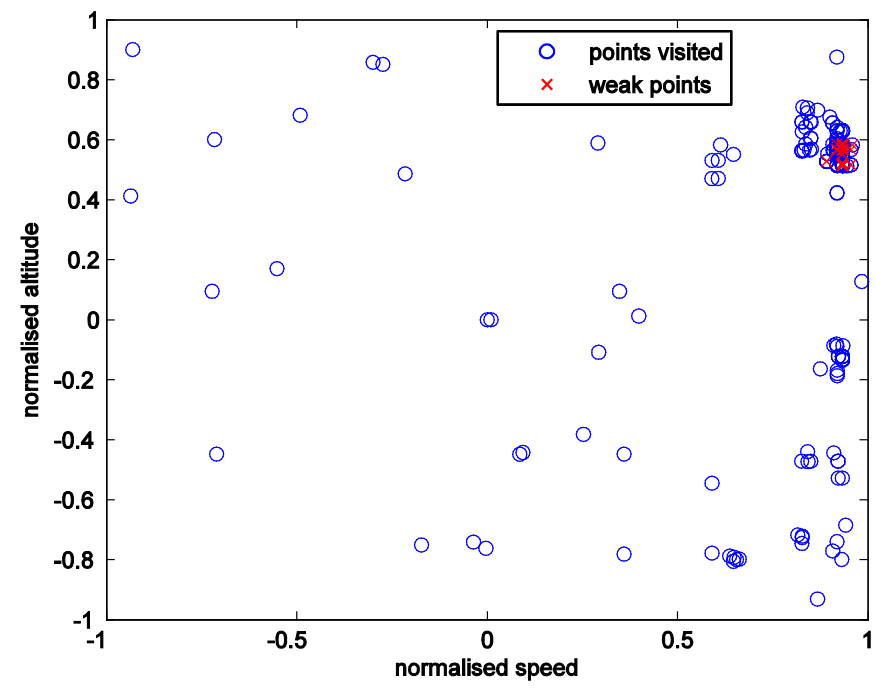

Fig. 3: WCS results indicating points in the normalized flight envelope with weak performance (x) regarding load factor limits of an experimental FCS applying an implementation of a genetic algorithm without niching concepts. 


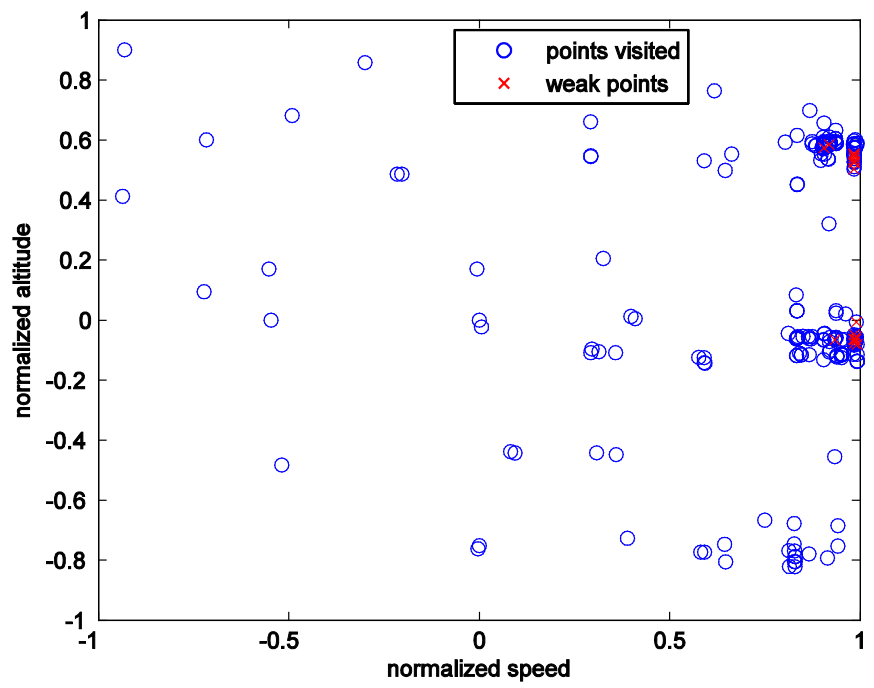

Fig. 4: WCS results indicating points in the normalized flight envelope with weak performance $(x)$ regarding load factor limits of an experimental FCS; application of a genetic algorithm including niching concepts. Two disconnected regions of noncompliance can be detected.

\section{Conclusions}

From these observations and results obtained for the benchmark problems, one can conclude that multi-objective optimization-based approaches can be a valuable part of assessment analysis procedures. While several criteria can be examined in one step, the computational effort does not increase compared to single objective assessment. Furthermore, the detection whether several assessment criteria can be violated simultaneously can only be made by multi-objective considerations.

The concepts of norm minimization with assessment constraints and the niching concept can be effectively used to find regions of noncompliance or to provide information about regions of safe operation. Moreover, the knowledge of parameter effects on assessment criteria can give useful advice for designing a FCS.

The direct formulation of assessment criteria from simulation results can lead to noisy or even discontinuous objective functions with multiple minima which are difficult to detect. However, genetic search techniques are insensitive to such kind of disturbed criteria and hence are able to solve the worst case search effectively and reliably.

Optimization based worst case search seems to be very 'aggressive' in the sense that it can take advantage out of any possibly incomplete or incorrect model- 
ling of the validation criterion. Hence, unsatisfactory criterion values are not necessarily due to control law weaknesses only. Before identifying a criterion not to be cleared the preconditions have to be verified carefully such as: validity of parameter ranges; completeness and correctness of the models involved (aircraft, controller, criterion); reasonable and realistic maneuvers and according implementations as simulation tasks. Since worst case search often operates at extreme flight conditions in the flight envelope, the validity of the aerodynamic models in those regions is of great importance. Therefore worst case search can not only be applied for assessment of flight control laws but also for verification of design and simulation models.

\section{References}

1. Dep, K., Pratrap, A., Agarwal, S and Meyarivan, T., “A Fast and Elitist Multiobjective Genetic Algorithm: NSGA-II”, IEEE Transactions on Evolutionary Computation, Vol.. 6, No. 2, 2002

2. Fielding, C., A. Varga, S. Benani and M. Sellier, (ed.), Advanced Techniques for Clearance of Flight Control Laws, Vol. 283 of Lecture Notes in Control and Information Science, Springer-Verlag, Berlin, 2002.

3. Fonseca, C.M., Fleming, P.J., “An overview of evolutionary algorithms in multiobjective optimization”, Evolutionary Computing., Vol. 3, No. 1, pp. 1-16, 1995.

4. Forssell, L. S., "Flight clearance analysis using global nonlinear optimization-based search algorithms”, AIAA Guidance, Navigation, and Control Conference and Exhibit, 2003, Austin, Texas, pp. 1023-1030.

5. David E. Goldberg and J. Richardson, Genetic Algorithms with sharing for multimodal function optimization, In J. J. Grefenstette, Proceedings of the Second International Conference on Genetic Algorithms, pages 41--49, Lawrence Erlbaum Associates, Hillsdale, NJ, 1987

6. Joos, H.-D., Bals, J., Looye, G., Schnepper, K., Varga, A., “A multi-objective optimization based software environment for control systems design”, Proceedings of the 2002 IEEE International Conference on Control Applications and International Symposium on Computer Aided Control Systems Design, CCA/CACSD, Glasgow, Scotland, U.K., 2002.

7 Joos, H.-D., “Worst Case Parameter Search Based Clearance Using Parallel Nonlinear Programming Methods", Optimization Based Clearance of Flight Control Laws, edited by A. Varga, A. Hansson and G. Puyou, Vol. 416 of Lecture Notes in Control and Information Science. Springer-Verlag, Berlin, 2011, pp. 149-158.

8. Marcos, Andres und Roux, Christophe und Rotunno, Max und Joos, Hans-Dieter und Bennani, Samir und Penin, Luis Felipe und Caramagno, Augusto (2011) The V\&V problematic for launchers: current practise and potential advantages on the application of modern analysis techniques. In: Proceedings of ESA GNC, 2011. 8th International ESA Conference on Guidance, Navigation \& Control Systems, Karlovy Vary, Czech Republic, 2011.

9. Menon, P. P., Kim, J., Bates, D. G., and Postlethwaite, I., “Clearance of nonlinear flight control laws using hybrid evolutionary optimization”,. IEEE Transactions on Evolutionary Computation, Vol. 10, No. 6, Dec. 2006, pp. 689-699.

10. Menon, P.P., Bates, D., and Postlethwaite, I., “Optimization-based flight control law clearance”, Nonlinear analysis and synthesis techniques for aircraft control, edited by D. Bates and D. M. Hagström, Vol. 365 of Lecture Notes in Control and Information Science. Springer-Verlag, Berlin, 2007. 
11. Menon, P.P., Bates, D., and Postlethwaite, I., "Nonlinear Robustness Analysis of Flight Control Laws for Highly Augmented Aircraft”, Journal of Control Engineering Practice, Vol. 15, No.6, pp. 655-662, 2007.

12. Pfiffer, H., and Joos, H.-D., "Robust Flight Control System Design Verification and Validation by Multiobjective Worst-case Search", AIAA GNC/AFM/MST/ASC, Minneapolis, USA, 2012

13. Varga, A., "Optimization-based Clearance", Advanced Techniques for Clearance of Flight Control Laws, edited by C. Fielding, A. Varga, S. Benani and M. Sellier, Vol. 283 of Lecture Notes in Control and Information Science. Springer-Verlag, Berlin, 2002.

14. Varga, A., Hansson, A., Puyou, G. (ed.), Optimization Based Clearance of Flight Control Laws, Vol. 416 of Lecture Notes in Control and Information Science. Springer-Verlag, Berlin, 2011.

15. Varga, A., Ossmann, D., and Joos, H.-D., "A fault diagnosis based reconfigurable longitudinal control system for managing loss of air data sensors for a civil aircraft", IFAC 2014 World Congress, Cape Town, South Africa.

16. Sawaragi, Y, Nakayama, H., and Tanino, T., “Theory of Multiobjective Optimization”, Academic Press Inc., Mathematics in Science and Engineering, Volume 176, Orlando Florida, 1985.

17. Zitzler, E., "Evolutionary Algorithms fo Multiobjective Optimization: Methods and Applications”, PhD thesis, Swiss Federal Institute of Technology (ETH), Zurich, Switzerland, November 1999. 